\title{
Holographic quantum mechanics
}

\author{
Kuyukov Vitaliy \\ vitalik.kayukov@mail.ru
}

SFU, Russia

27 oct 2019

\begin{abstract}
In this paper, we consider the interpretation of quantum mechanics based on the holographic principle. A simple analysis gives the following result. Particle energy is proportional to the density of holographic information in space. The particle momentum is proportional to the flow of holographic information. The density of holographic information reflects the wave properties of the particle.
\end{abstract}

In quantum mechanics, there are many interpretations about the wave properties of particles. Previously, David Bohm developed the idea of a holographic reality of the space of the Universe. This idea was reflected in the holographic principle in the works of T Hooft, Sasskind, Bekenstein, Hawking. Hawking's main discovery was to predict the temperature of a black hole

$$
T_{H}=\frac{c^{3} h}{8 \pi k G M}
$$

This means a black hole emits particles with energy

$$
E=k T_{H}=\frac{c^{3} h}{8 \pi G M}=\frac{c h}{4 \pi r_{g}}
$$

On the other hand, the surface of a black hole is proportional to the holographic information

$$
I_{\max }=\frac{c^{3} A}{4 G h}
$$

This is the holographic principle.

When a black hole emits a particle, its surface and volume decreases, and information also decreases

$$
\begin{gathered}
\Delta V=4 \pi r^{2} \Delta r=\frac{r}{2} \Delta A \\
\Delta I_{\max }=\frac{c^{3} \Delta A}{4 G h}=\frac{c^{3} \Delta V}{2 G h r}
\end{gathered}
$$

The result is a connection between the particle energy, holographic information and the size of the space.

$$
E=\frac{c h}{4 \pi r_{g}}=\frac{G h^{2}}{2 \pi c^{2}} \frac{\Delta I_{\max }}{\Delta V}
$$


Here we consider this formula as a confirmation of the holographic reality of the space of the Universe and quantum mechanics.

Particle energy is proportional to the density of holographic information

$$
E=m c^{2}=h \omega=\frac{G h^{2}}{2 \pi c^{2}} \frac{\Delta I_{\max }}{\Delta V}
$$

Maximum holographic information is limited by the number of hidden degrees of freedom of space.

$$
\Delta N=\Delta I_{\max }
$$

In addition, you can see that the frequency is proportional to the density of holographic information, which corresponds to the wave nature of holography

$$
\omega=\frac{G h}{2 \pi c^{2}} \frac{\Delta I_{\max }}{\Delta V}
$$

Hence, the particle momentum is associated with the density of the flow of holographic information

$$
p=\frac{E v}{c^{2}}=h k=\frac{G h^{2}}{2 \pi c^{4}} v \frac{\Delta I_{\max }}{\Delta V}
$$

Where the density of information flow is defined as

$$
J_{\max }=v \frac{\Delta I_{\max }}{\Delta V}
$$

As a result, the wave vector is proportional to the density of the flow of holographic information

$$
k=\frac{G h}{2 \pi c^{4}} J_{\max }
$$

The wave properties of a particle are related to the density of holographic information and indirectly confirms the idea of holographic reality

$$
\omega=\frac{G h}{2 \pi c^{2}} \frac{\Delta I_{\max }}{\Delta V}
$$

This is an interesting result; wave nature has duality with geometry and holographic information.

This work once again confirms the holographic reality of the David Bohm Universe in the form of the density of holographic information. 
[1]. Roger Penrose, Foreword: A Computable Universe, Understanding Computation and Exploring Nature As Computation, arxiv: 1205.5823, 2012.

[2] L. Nottale, Fractal spacetime and microphysics, World Scientific, 1993

[4] H. Ohanian and A. Ruffini, Gravitation and spacetime, Norton, 1994

[5] L. Olavo, Physica A, 262 (1999), 197-214 and 271 (1999), 260-302 and 303-323; Phys. Rev. E, 64 (2001), 036125

[6] R. Parwani, quant-ph 0408185, 0412192, and 0508125

[7] N. Pinto-Neto, gr-qc 0410001, 0410117, and 0410225

[7] N. Pinto-Neto and E. Santini, gr-qc 0009080 and 0302112; Gener. Relativ. Gravitation, 34 (2002), 505; Phys. Lett. A, 315 (2003), 36; Phys. Rev. D, 59 (1999), 123517 (gr-qc 9811067)

[8] S. Popescu, A. Short, and A. Winter, quant-ph 0511225

[9] C. J. Isham, "Topological and global aspects of quantum theory," in Relativ. Groups Topol. II (B. S. DeWitt and R. Stora, eds.). North-Holland, Amsterdam, 1984.

[10] Josef Kluson, "Canonical Analysis of Inhomogeneous Dark Energy Model and Theory of Limiting Curvature," arXiv:1701.08523 *hep-th].

[11] H. Firouzjahi, M. A. Gorji, and A. H. Mansoori, "Instabilities in Mimetic Matter Perturbations," arXiv:1703.02923 [hep-th].

[12] S. Hojman, K. Kuchar, and C. Teitelboim, "Geometrodynamics regained," Annals of Physics 96 (1976) 88-135. 